\title{
Postural Behaviour Responses to Visual Stimulation in Patients with Vestibular Disorders
}

\author{
H. SUAREZ ${ }^{1}$, P. MUSE ${ }^{2}$, A. SUAREZ ${ }^{1}$ and M. AROCENA ${ }^{1}$ \\ From the ${ }^{1}$ Laboratory of Audiology and Vestibular Pathophysiology and the ${ }^{2}$ Department of Biomedical Engineering, School of Medicine, \\ Montevideo, Uruguay
}

\begin{abstract}
Suarez H, Muse P, Suarez A, Arocena M. Postural behaviour responses to visual stimulation in patients with vestibular disorders. Acta Otolaryngol 2000; 120: 168-172.

Patients with different vestibular disorders exhibit changes in postural behaviour when they receive visual stimuli, reproducing environmental stimulation. Postural control was studied using an AMTI Accusway platform, measuring the confidential ellipse (CE) and sway velocity (SV). Postural responses were recorded according to the following stimulation paradigm: $i$ ) without specific stimuli; ii) smooth pursuit with pure sinusoids of $0.2 \mathrm{~Hz}$ (foveal stimulation); and iii) optokinetic stimulation (retinal stimuli). Patients with central vestibular disorders (CVD), cerebellar damage and unilateral peripheral vestibular lesions (UPVL) in asymptomatic periods were studied. A group of normal subjects was studied as control. Signal processing was done with a scalogram by wavelets in order to observe the relation between time and frequency in postural control. While patients with CVD and cerebellar disease showed a significant increase in CE and SV in the three conditions of the paradigm compared to the normal group, the patients with UPVL showed no change. Wavelets processing showed that the main sway occurs in the $\mathrm{Y}$ axis (antero-posterior) and below at $0.4 \mathrm{~Hz}$ in normal subjects, while the CVD and cerebellar patients showed sway frequencies in both the $\mathrm{X}$ and $\mathrm{Y}$ axes. The clinical implications of these findings are discussed. Key words: olivo-ponto-cerebellar atrophy, optokinetic stimulation, posturography, smooth pursuit, vestibular disorders.
\end{abstract}

\section{INTRODUCTION}

Although the influences of sensory cues on postural sway have been demonstrated (1), less information is available about how visual, somatosensory and vestibular motion information is combined and processed by the central nervous system (CNS) for postural control (2). Some models describing this interaction have been developed. As various components of the postural system are non-lineal, simple behavioural explanations are avoided. Thus, if the body is modeled as a multi-link structure, the equations of motion become very complex (3). However, it is necessary to have valid measures to assess postural control in clinical settings, since changes in visual surroundings elicit balance disorders and falls in patients with vestibular disorders. Because visual feedback is used as a tool in vestibular rehabilitation, it would be useful to have quantitative measures of postural control to assess therapy outcomes (4).

The multicomponent nature of imbalance in patients with CNS damage has been described and measured by means of posturography (5), but a more detailed study of postural responses generated by some specific visual stimulation would provide more information about how changes in the visual field are involved in the mechanisms of instability and fall. In fact, visual stimulation can be foveal and voluntary (smooth pursuit), or retinal and non- voluntary (optokinetic stimulation), triggering different neural networks to maintain a stable visual field on the retina.

Our goal was to assess the postural responses when patients with vestibular disorders are exposed to different visual stimuli. Behaviour in postural control when patients are exposed to different kinds of visual stimulation was tested in three clinical models: $i)$ patients with central vestibular disorders (CVD) and normal cerebellar function; ii) patients with CVD and cerebellar damage; and iii) patients with unilateral vestibular disease.

\section{METHODS}

Postural behaviour responses to visual stimuli were analysed in three different pathological groups. The group with CVD and normal cerebellar functions included 12 patients (69-82 years old) who had had more than two falls in a year and who manifested central vestibular alterations registered by electronystagmography (ENG). The second group included patients with CVD and cerebellar damage. Nine patients with olivo-ponto-cerebellar atrophy (23-47 years old), diagnosed according to Harding's criteria (6), were chosen. Initial clinical manifestations of the disease had appeared within three years of the study. The third group included patients with unilateral peripheral vestibular disorders (UPVL). This population was represented by nine patients 
(34-59 years old) with unilateral Meniere's disease and recurrent vertigo with unilateral vestibular hypoactive function, registered in periods without symptoms.

A group of 24 volunteers (21-59 years old) was used as control. All patients were assessed with ENG, audiological testing and magnetic resonance images or computerized axial tomography. This protocol was applied after full informed consent had been received from all the subjects.

The stimulation paradigm was as follows:

1. Subjects were in a standing position, eyes open, without stimulation.

2. Foveal stimuli were applied to pure sinusoids at $0.2 \mathrm{~Hz}$ ( $1 \mathrm{~m}$ from the lead's bar).

3. Retinal stimuli were applied optokinetically (OK) at $65^{\circ} / \mathrm{s}$ angular velocity.

During OK stimulation, postural responses were measured using an AMTI Accusway platform with online recording of the centre of pressure (COP), measuring two relevant parameters: sway velocity and confidential ellipse.

\section{Sway velocity}

An 80 -s trial was recorded, leading to two discrete signals of $N=4,000$ samples (sampling frequency $\mathrm{fs}=50 \mathrm{~Hz}$ ), COP $x$ and COPy. Then, for each record, the average speed of COP along its path $(\langle v\rangle)$ was calculated at $\mathrm{t}=10 \mathrm{~s}(N=500)$ and $\mathrm{t}=80 \mathrm{~s}(N=$ 4,000) using:

$$
\begin{gathered}
<v>=\frac{f_{s}}{N} \sum_{i=2}^{N}\left[\left(C O P x_{i}-C O P x_{i-1}\right)^{2}+\left(C O P y_{i}-\right.\right. \\
\left.\left.C O P y_{i-1}\right)^{2}\right]^{1 / 2}
\end{gathered}
$$

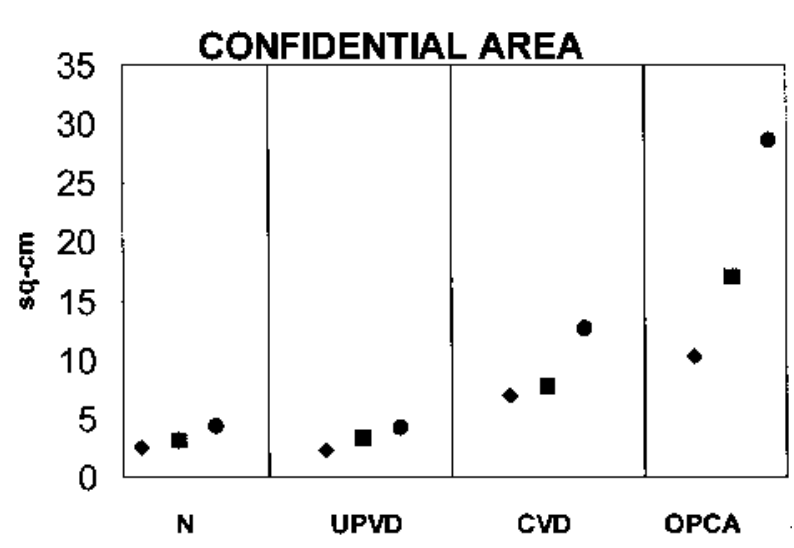

Fig. 1. CE values in $\mathrm{N}$ (normal group), UPVL patient (Meniere's disease in asymptomatic period), CVD patient (representative patient with CVD) and OPCA (olivo-pontocerebellar atrophy) patient. Stimulation paradigm conditions $1(\diamond), 2(\boldsymbol{\square})$ and $3(\bullet)$.

\section{Confidential ellipse}

The 95\% confidence ellipse of the bivariate distribution $\left(C O P x_{i}, C O P y_{i}\right), 1 \leq i \leq N$, is the ellipse where $95 \%$ of the COP's samples are expected to be enclosed. It can be shown that the area of the $95 \%$ confidence ellipse is:

Area $=2 \pi F_{0.05[2, N-2]} \sqrt{\sigma_{x}^{2} \sigma_{y}^{2}-\sigma_{x y}^{2}}$

where $F_{0.05[2, N-2]}$ is the $F$ statistic at a confidence level of 0.95 with $\mathrm{N}$ data points, $\sigma_{x}^{2}$ and $\sigma_{y}^{2}$ are the variances of the ML and AP coordinates, respectively, and $\sigma_{x y}$ is the covariance. For a large sample size $(N>120), F_{0.05[2, N-2]}$ is 3.00 . This is our case, since we calculate the $95 \%$ confidence ellipses of 80 -s registers $(N=4,000)$.

\section{Time frequency analysis: scalogram}

In order to evaluate the sway frequency contents and their temporal behaviour, a time frequency analysis of COP in both directions (COPX and COPy) was performed including computation of its scalogram. Since the Fourier transformation is not adapted to the analysis of non-stationary signals like the COP signal, it must be considered its time-frequency representation. An often used time frequency energy density, because of its resolution properties, is the scalogram (7). The scalogram of a signal $x(u)$ is the energetic version of the wavelet transformation (WT), defined as the square magnitude of the WT:

$S C A L_{x}(t, f)=\left|\int_{-\infty}^{+\infty} x(u) \cdot \sqrt{\frac{f}{f_{0}}} \cdot \psi *\left(\frac{f}{f_{0}} \cdot(u-t)\right) \cdot d u\right|^{2}$

The mother wavelet $\psi(u)$ that was chosen was the Morlet wavelet (8):

$\psi(u)=e^{-u^{2} / 2} \cdot e^{j 2 \pi f_{0} u}$

This wavelet has the best time-frequency localization in the sense specified by the Heisemberg-Gabor uncertainty principle.

Significant changes in the CE and SV were determined using Student's $t$-test for independent samples, and as level of significant of error, an alpha $\leq 0.05$ was accepted.

\section{RESULTS}

Patients with CVD and those with cerebellar damage showed significant increments in sway velocity (SV) in the three conditions of the stimulation paradigm (Fig. 2, Table I). Similar behaviour was observed with the $\mathrm{CE}$, where the COP distribution showed a significant increment in patients with CVD and those with cerebellar damage. This last group also showed 
Table I. Average values and standard deviations in the three paradigm stimulation conditions (1,2 and 3) in the normal group and in UVPL, CVD and OPCA patients.

\begin{tabular}{|c|c|c|c|c|c|c|}
\hline \multirow[b]{2}{*}{ Condition } & \multicolumn{3}{|c|}{ Confidential ellipse $\left(\mathrm{cm}^{2}\right)$} & \multicolumn{3}{|c|}{ Sway velocity $(\mathrm{cm} / \mathrm{s})$} \\
\hline & 1 & 2 & 3 & 1 & 2 & 3 \\
\hline Normal subjects & $2.42 \pm 0.50$ & $3.22 \pm 1.27$ & $4.21 \pm 1.77$ & $1.31 \pm 0.16$ & $1.33 \pm 0.18$ & $2.04 \pm 0.62$ \\
\hline UPVL & $1.83 \pm 0.44$ & $3.32 \pm 0.49$ & $3.73 \pm 0.45$ & $1.48 \pm 0.22$ & $1.57 \pm 0.36$ & $2.50 \pm 1.05$ \\
\hline CVD & $8.78 \pm 1.27$ & $8.20 \pm 1.58$ & $8.70 \pm 2.86$ & $3.21 \pm 1.01$ & $3.02 \pm 0.27$ & $3.89 \pm 1.15$ \\
\hline OPCA & $12.05 \pm 2.09$ & $17.31 \pm 4.82$ & $15.74 \pm 4.71$ & $4.64 \pm 0.75$ & $4.17 \pm 0.86$ & $3.28 \pm 0.64$ \\
\hline
\end{tabular}

a much higher increase in the COP area in the three conditions of the paradigm (Fig. 1, Table I). UPVL patients had similar measures of CE and SV compared to the normal group when recorded in periods without vertigo.

In normal subjects, wavelets processing allowed us to see that the sway frequency content was mainly in the $\mathrm{Y}$ axis (antero-posterior) in frequencies bellow $0.4 \mathrm{~Hz}$ in all conditions of the stimulation paradigm. In patients with CVD and in cerebellar patients, the frequency content was distributed in both the $\mathrm{X}$ and $\mathrm{Y}$ axes with visual stimulation (Fig. 3).

\section{DISCUSSION}

In recent years, various parameters to quantify postural dysfunction have been intensely studied. It is important also to evaluate how somatosensory, vestibular and visual information modifies postural behaviours. The modifications to postural control produced by foveal and retinal visual information in the three different populations were studied.

Results clearly show that foveal or retinal information provokes postural behaviour changes, increasing the SV and COP distribution area (CE) in patients with CVD, especially in the ones with cerebellar damage, while in the normal group and UPVL patients, the changes were not significant.

Visual stimulation, such as that used in this paradigm, reproduces similar environmental stimulation. It is very important to reproduce these situations in order to improve our knowledge of how visual stimuli can trigger instability in patients with vestibular disorders. The data suggest that $\mathrm{CE}$ and SV are valid parameters to assess modifications in postural behaviour in vestibular patients, and also to determine when certain kinds of visual information generate a significant increase in postural disability and fall risk, specially in elderly people with CVD or patients with cerebellar disorders.
The UPVL patients in the asymptomatic period adjusted their postural control when they received visual stimulation in a way similar to that in the normal group (Figs 1 and 3). The scalogram revealed that in the normal group, most of the sway frequency content was in the $\mathrm{Y}$ axis, and the posture adjusted after visual stimulation in the first 20 s. However, in the CVD and cerebellar patients, the sway frequency content was significant in both axes with an antero-posterior and lateral sway with no adaptation behaviour during the $80 \mathrm{~s}$ of recording (Fig. 3).

Therefore, changes in postural behaviour in CVD and cerebellar patients after visual stimulation occur with high sway velocity and increases in the COP distribution (CE) in both axes (lateral and anteroposterior). These findings are important for planning rehabilitation therapy.

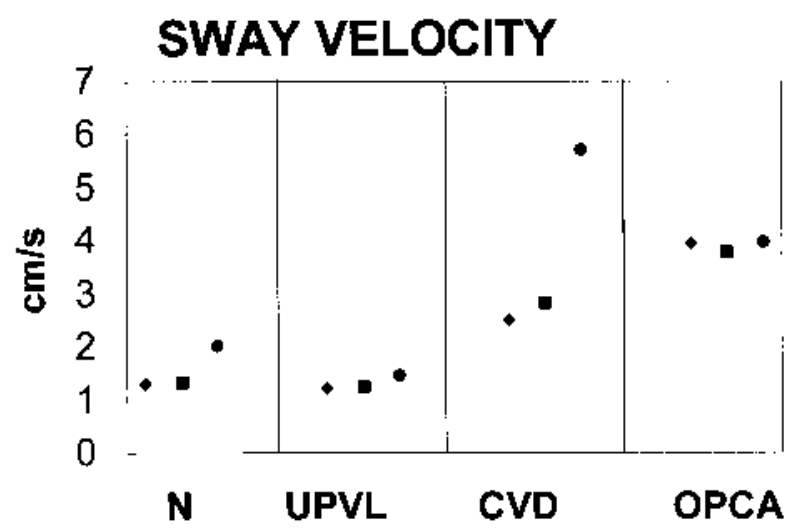

Fig. 2. SV values in $\mathrm{N}$ (normal group), UPVL patient (Meniere's disease in asymptomatic period), CVD patient (representative patient with CVD) and OPCA (olivo-pontocerebellar atrophy) patient. CVD patient shows the higher $\mathrm{SV}$ value in response to $\mathrm{OK}$ stimulation. Stimulation paradigm conditions $1(\bullet), 2(\boldsymbol{\square})$ and $3(\bullet)$. 


\section{NORMAL SUBJECT}

SCALO, Morlet wevelet, $N h 0=12.65, N=256$, lin. scale. Thld $=5 \%$

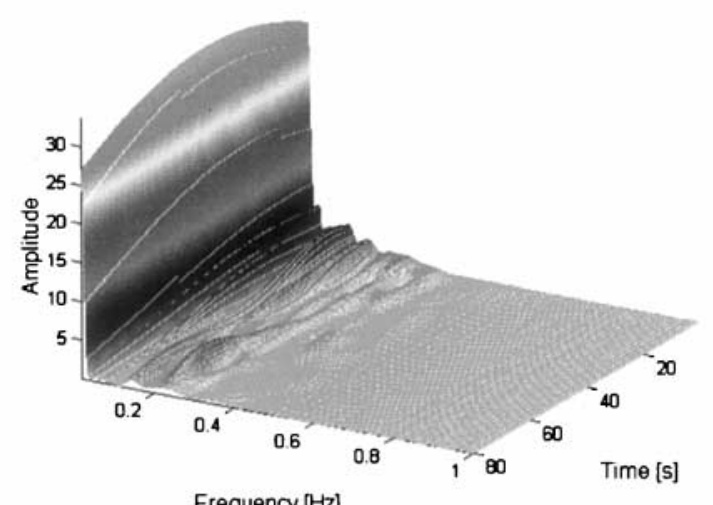

A
SCALO, Morlet wavelet, $N h 0=12.65, N=256$, lin. scale, Thld $=5 \%$

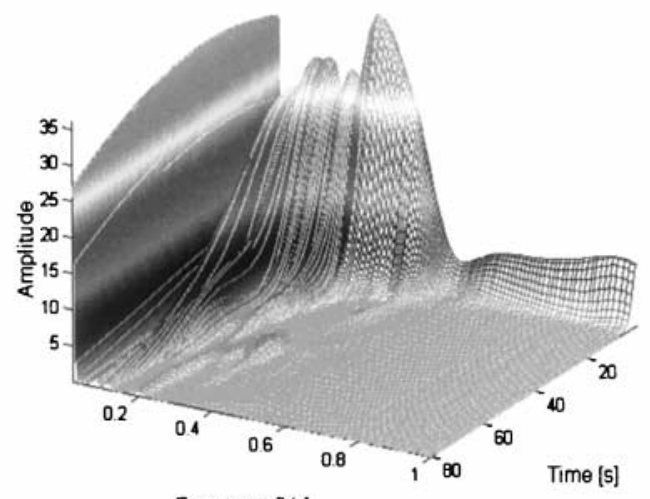

B

\section{CVD}
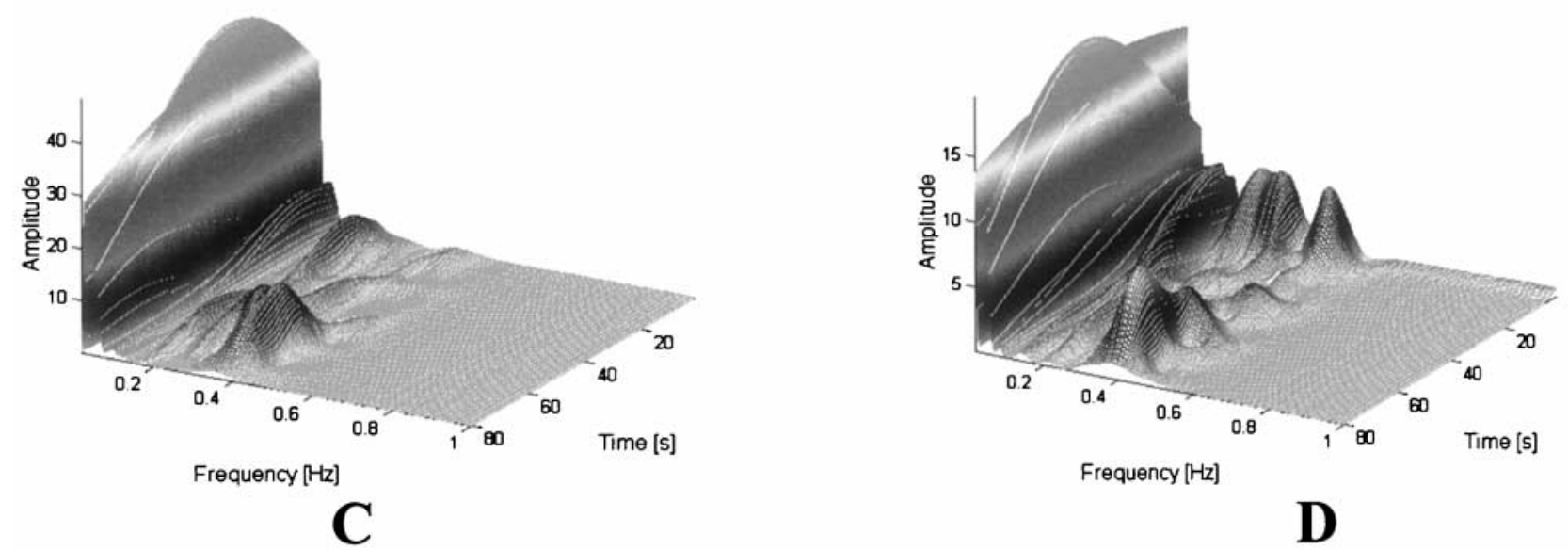

D

Fig. 3. Scalogram of the COP distribution with foveal stimulation. Representative normal subject: $(A)$ COP distribution in the $\mathrm{X}$ axis and $(B)$ COP distribution in the $\mathrm{Y}$ axis. Representative patient with CVD: $(C)$ COP distribution in the $\mathrm{X}$ axis and $(D)$ COP distribution in the Y axis. The normal subject with foveal stimulation "adjusted" his postural response in the first $20 \mathrm{~s}$ mainly in the $\mathrm{Y}$ axis (anteroposterior sway) with frequencies below $0.4 \mathrm{~Hz}$. The CVD patient shows a distribution of the sway frequencies content in both axes during the $80 \mathrm{~s}$.

\section{REFERENCES}

1. Nashner LM. Neurophysiology of the balance system and dynamic platform: posturography. In: Arenberg K, ed. Dizziness and balance disorders. Amsterdam/New York: Kluger, 1993: 363-7.

2. Brandt TH. Sensory function and posture. In: Amblard B, Berthoz A, Clarc F, eds. Posture and gait: development, adaptation and modulation. Amsterdam: Elsevier, 1988: $127-36$.
3. Peterka RJ. Simple model of sensory interaction in human postural control. In: Mergner T, Hlavacka F, eds. Multisensory control of posture. New York/London: Plenum Press, 1995: 281-8.

4. Suarez H, Arocena M. Clinical assessment and balance training in cerebellar patients. In: Arenberg K, ed. Dizziness and balance disorders. Amsterdam/New York: Kluger, 1993: $737-45$.

5. Allum JHJ. Posturographic system: current measurement concepts and possible improvement. In: Brandt T, 
Paulus W, Bles W, Dietrich M, Krafesyk S, Straube A, eds. Disorders of posture and gait. Stuttgart/New York: George Thieme Verlag, 1990: 16-28.

6. Harding AE. The hereditary ataxia and related disorders. Clinical neurology and neurosurgery monographs. Vol. 6. London: Churchill-Livingstone, 1984.

7. Rioul O, Vetterli M. Wavelets and signal processing. IEEE Signal Processing Magazine 1991; 14-38.

8. Kronland-Martinet J, Morlet J, Grossman A. Analysis of sound patterns through wavelet transforms. Interna- tional Journal of Pattern Recognition and Artificial Intelligence 1987; 1: 273-301.

Address for correspondence:

Dr. H. Suarez

Laboratory of Audiology and Vestibular Pathophysiology

C. de Guayaquil 1332 Montevideo

11400 Uruguay

Fax: (5982) 48740 80,

E-mail: hsuarez@chasque.apc.org 